\title{
PHILIPPE HAMMAN
}

\section{FIGURES DE L'ÉTAT DANS L'ENTRE-DEUX: LE REICHSLAND D'ALSACE-LORRAINE, »LABORATOIRE« D'UNE POLITIQUE DE GERMANISATION ENTRE LA FRANCE ET L'ALLEMAGNE (1871-1918)}

\author{
"Das Reichsland Elsaß-Lothringen« \\ Une construction politique contingente
}

En 1871, peu après la fondation du Reich et la signature du traité de Francfort qui consacre le transfert de souveraineté de l'Alsace-Lorraine, Bismarck fait voter par le Reichstag une forme d'organisation du territoire conquis sous le nom de »Reichsland Elsaß-Lothringen«. Cette terre d'Empire établie à partir des anciens départements français de Moselle, du Bas-Rhin et du Haut-Rhin est une novation, façonnée en fonction des événements, une "construction politico-administrative sinachevée «' qui permet de suivre au plus près les processus en train de se faire entre France et Allemagne. Dès l'origine, les contingences politiques et diplomatiques se révèlent dans toute leur complexité: faire de l'Alsace-Lorraine une propriété commune des États allemands confédérés doit permettre d'éviter d'une part l'annexion directe à la Prusse sur le modèle de la province rhénane, ce qui romprait l'équilibre des vainqueurs (Bavière, Bade...), et de l'autre le partage du territoire entre ces mêmes États, forcément sujet à polémique dans son tracé concret. La loi d'Empire du 9 juin 1871 instaure le Reichsland, bientôt complétée par la loi du 30 décembre 1871 sur l'organisation administrative de l'Alsace-Lorraine. Une forme juridique nouvelle s'impose, en même temps que les termes retenus traduisent l'inédit jamais auparavant l'Alsace et la Lorraine n'avaient été administrées ensemble - et ses embarras: la solution du Reichsland émerge dans la mesure où l'Allemagne est devenue un empire, mais on parle de »Land« et non de »Staat« avec toute la part d'indéfini que cela révèle.

À grands traits, on peut présenter le statut politique du Reichsland qui découle de ces circonstances autour de quelques principes, parfois résumés dans l'expression »dictature impériale« (Kaiserdiktatur), qui montre que la terre

' Selon l'expression de François ROTH, Lorraine, France, Allemagne. Un parcours d'historien, Metz 2002, p. 51. 
d'Empire n'est pas placée au même niveau que les États confédérés du Reich; l'autonomie inteme serait contraire au projet germanisateur' ${ }^{2}$ :

- Le pouvoir d'État (Staatsgewalt) y est exercé par l'Empereur au nom des États confédérés.

- Le pouvoir politique est dévolu au chancelier impérial, qui l'exerce d'abord en s'appuyant sur un office spécialisé à Berlin, le »Reichskanzleramt für Elsaß-Lothringen«, puis via un gouverneur installé à Strasbourg à compter de 1879.

- Le pouvoir législatif relève dans un premier temps de l'Empereur puis, à compter de 1874, du Reichstag à Berlin (ce qui n'exclut pas le maintien d'une part de législation française antérieure: par exemple, la législation scolaire, les conseils généraux...).

- Le pouvoir administratif, enfin, est confié à des hauts fonctionnaires allemands, qui se voient attribuer simultanément deux missions, entre fonction réglementaire et politique: appliquer les nouvelles règles de droit et assurer l'irréversibilité des »faits accomplis« de l'annexion.

Afin d'appréhender les figures de l'État entre la France et l'Allemagne, cette construction historique originale qu'a été le Reichsland d'Alsace-Lorraine de 1871 à 1918 permet ainsi d'approcher, suite à une rupture politique et militaire, l'arrivée d'une nouvelle administration allemande en zone annexée. De la sorte, on analyse de façon affinée les discours courants sur la germanisation de ce territoire, en s'intéressant aux incarnations de l'État allemand en terre d'Empire. Pour ce faire, on se propose d'interroger la production de nouveaux périmètres étatiques et découpages administratifs dans leur relation avec l'apparition de nouveaux acteurs de l'État dans ces espaces remodelés: pour questionner la promotion d'élites »importées«, entre continuité administrative et renouvellement, bureaucratie et politisation, on focalisera sur le profil et l'action des fonctionnaires mis en place aux différents échelons de l'intervention publique; ceci permettra de saisir sur quels personnels l'Empire s'appuie pour pénétrer le territoire conquis, quelles possibilités de carrière apparaissent ici, et en même temps quelles contradictions au sommet de l'État expliquent les soubresauts locaux, s'agissant d'organiser l'inédit et de gérer l'entre-deux. Ces configurations rendent raison des trajectoires des acteurs et des dynamiques institutionnelles engagées, dont on ne saurait postuler la linéarité, mais qui, tout au contraire, à travers leurs routines, leurs accélérations et leurs crises, nous renseignent sur les répercussions des relations interétatiques dans ce "laboratoire« original de la germanisation ${ }^{3}$.

2 Sur le cadre juridique, Robert REDSLOB, Le régime politique de l'Alsace-Lorraine sous la domination allemande, dans: Revue de droit public et de sciences politiques (1921) p. 5-63.

${ }^{3}$ Cet article prolonge notre thèse de science politique: Les transformations de la notabilité: l'industrie faïencière à Sarreguemines (de 1836 à nos jours), Institut d'études politiques, Strasbourg 2000, $3^{\mathrm{e}}$ partie, publiée sous forme remaniée: Philippe HAMMAN, Les transforma- 
L'invention d'une administration ou comment organiser l'inédit

Avant de focaliser sur ses acteurs et ses spécificités liées à l'entre-deux, on rappellera brièvement comment se présente la structure administrative du Reichsland, en distinguant le régime de la Présidence supérieure de celui du Statthalterat.

\section{La Présidence supérieure (1871-1879)}

Si l'annexion pure et simple à la Prusse n'a pas été retenue par Bismarck, c'est bien le "modèle« administratif prussien ${ }^{4}$ qu'il introduit en Alsace-Lorraine en 1871, insérant les trois départements français conquis et remodelés dans une Présidence supérieure déjà expérimentée en province rhénane voisine, avec pour conséquence les lourdeurs de la partition du pouvoir décisionnel entre Berlin et Strasbourg: certains domaines sont de la compétence directe du Reich, comme les chemins de fer et les affaires militaires, et de façon générale le Reichstag édicte la loi, tandis que l'administration prend place à Strasbourg mais sous une véritable tutelle de Berlin. Par ailleurs, afin de rallier les notables locaux, est instauré un parlement régional ou "Délégation « (Landesausschuß), élu au deuxième degré sur la base des conseils généraux et doté de prérogatives encadrées par les autorités ${ }^{5}$. Ce schéma complexe ne dure guère en l'état, s'effaçant au profit de la "Constitution de 1879 «, marquée, elle, par la stabilité, puisque globalement en vigueur jusqu'en 1918.

tions de la notabilité entre France et Allemagne. L'industrie faïencière à Sarreguemines (1836-1918) Paris 2005. On renvoie également aux précieux travaux d'historiens, dont François ROTH, La Lorraine annexée. Étude sur la Présidence de Lorraine dans l'Empire allemand, Nancy 1976; Hermann HIERY, Reichstagswahlen im Reichsland. Ein Beitrag zur Landesgeschichte von ElsaB-Lothringen und zur Wahlgeschichte des Deutschen Reiches. 1871-1918, Düsseldorf 1986; et la synthèse de Bernard VoGLER, Histoire politique de l'Alsace, Strasbourg 1995.

${ }^{4}$ Pour une étude de l'administration prussienne sous l'angle de ses acteurs, voir MarieBénédicte DAVIET-VINCENT, La prise en compte de plusieurs générations dans la méthode prosopographique: l'exemple des hauts fonctionnaires prussiens sous l'Empire et la république de Weimar, dans: Genèses. Sciences sociales et histoire 56 (2004) p. 117-130.

${ }^{5}$ Voir notamment Georg WOLFRAM, Oberpräsident Eduard von Möller und die elsaßlothringische Verfassungsfrage, Berlin 1925. 


\section{Le Statthalterat (1879-1918)}

\section{Le niveau du Reichsland}

Les modifications institutionnelles introduites par la loi du 4 juillet 1879 tiennent surtout à la nomination à Strasbourg d'un gouverneur (Statthalter), correspondant au transfert de la direction de l'administration civile du Reichsland dans la nouvelle capitale régionale et non plus à Berlin. Le Statthalter a rang de chancelier de l'Empereur et exerce par délégation le pouvoir de souveraineté, dont il rend compte directement à ce dernier (Immediatbericht). Il se voit adosser pour cela une administration à Strasbourg (Ministerium), à la tête de laquelle est placé un secrétaire d'État. Corrélativement, à l'exception des questions de souveraineté relevant toujours du Reichstag, la Délégation d'AlsaceLorraine voit ses pouvoirs de droit commun accrus, d'où l'apparition d'un espace juridique spécifique en même temps que d'une compétition politique régionale: la réforme assouplit le statut du Reichsland pour favoriser un particularisme censé permettre l'intégration à plus long terme. Pour autant, il ne s'agit pas d'un alignement sur les États confédérés: l'Empereur et le Bundesrat conservent en particulier un droit de veto législatif; c'est davantage de décentralisation administrative que l'on peut parler.

Or, au début du $\mathrm{XX}^{\mathrm{e}}$ siècle, nombreux sont les mouvements alsacienslorrains qui manifestent leur souhait de modifications constitutionnelles dans le sens de l'acquisition de l'autonomie et du statut d'État confédéré à part entière $^{7}$. En fait, suite aux mobilisations des partis politiques allemands, la réforme - parfois appelée »Constitution de 1911«-s'est centrée sur le remplacement de la Délégation, contrôlée par les notables régionaux, par un Landtag à deux chambres, dont une élue au suffrage universel direct. Pour le reste, les pouvoirs du Statthalter et de son administration sont maintenus, de même que la spécificité du Reichsland, qui demeure, selon la formule du juriste Robert Redslob, "un pays dépendant« (ein abhängiges Land) ${ }^{8}$. Quant aux années 1914-1918, la guerre induit un régime de dictature militaire en AlsaceLorraine?

\footnotetext{
${ }^{6}$ Voir Georg Wolfram, Verfassung und Verwaltung von Elsaß-Lothringen, 1871-1918, dans: Das Reichsland Elsaß-Lothringen. 1871-1918, Berlin 1936.

${ }^{7}$ Sur ces débats, voir Jean-Marie MAYEUR, Autonomie et politique en Alsace. La Constitution de 1911, Paris 1970; et Hermann HIERY, Zwischen Scylla und Charybdis: Carl Graf von Wedel als Statthalter im Reichsland Elsaß-Lothringen, dans: Zeitschrift für die Geschichte des Oberrheins (1986) p. 299-328.

${ }^{8}$ Robert REDSLOB, Unabhängige Länder, Leipzig 1914.

9 Voir le témoignage de l'abbé Émile Wetterlé, figure politique alsacienne: Ce qu'était l'Alsace-Lorraine et ce qu'elle sera, Paris 1915.
} 
La réorganisation des niveaux d'administration du territoire

Ces dynamiques d'évolutions institutionnelles se retrouvent aux échelons inférieurs d'administration de la terre d'Empire: les districts, cercles et communes. Une loi du 30 décembre 1871 fixe les contours des anciens départements transformés en trois districts (Bezirke) - Basse-Alsace, Haute-Alsace et Lorraine (qui correspond au département de Moselle reconfiguré) -, eux-mêmes divisés en cercles (Kreise). Si une loi du 24 janvier 1873 crée les »assemblées de district" (Bezirkstage) ou "de cercle" (Kreistage), qui reprennent pour l'essentiel les attributions françaises antérieures des conseils généraux et d'arrondissement, la filiation administrative est plus complexe.

À la tête du Bezirk, un président (Bezirkspräsident) succède au préfet français, mais ses compétences sont objet de discussions permanentes, tantôt réduites, tantôt rétablies: c'est que le président est en droit à la fois le représentant de l'État et l'exécutant des décisions des assemblées de district. Ses prérogatives, par rapport à l'ancien préfet, sont grignotées d'»en haut« et d'»en bas«. La présidence apparaît fréquemment non tant comme un centre de décision qu'un relais des instances de Strasbourg. Certaines attributions ont d'ailleurs été explicitement transférées au fil des années: l'enseignement secondaire (1872), les écoles normales primaires (1883) ou encore les contributions directes (1894). En 1914 est même posée ouvertement la question de la suppression des Bezirke, en raison des compétences croissantes exercées par le Landtag. Parallèlement, la loi municipale de 1895 confie aux responsables de cercles la tutelle des communes de moins de 2000 habitants. Cet encadrement des responsabilités administratives révèle la portée véritable de la fonction de Bezirkspräsident: titulaire du pouvoir hiérarchique sur les fonctionnaires des districts, il est aussi le responsable politique du Bezirk aux yeux des autorités de l'Empire - sa nomination relève du reste de l'Empereur, qui a exercé à plusieurs reprises ce choix personnellement ${ }^{10}$. La correspondance laissée par le président de Lorraine von Hammerstein, en fonction d'août 1883 à mars 1901, traduit bien cette difficulté du poste, notamment après l'installation de Guillaume II au château d'Urville en 1890: chaque séjour ou visite du Kaiser en Lorraine met les décisions administratives (et leur responsable) en péril si l'Empereur suit les requêtes formulées par divers élus et notabilités ${ }^{11}$.

Au niveau inférieur, les changements de structure sont très palpables: la loi du 30 décembre 1871 fractionne les anciens arrondissements français, jugés trop étendus, en des cercles deux fois plus nombreux: huit en Basse-Alsace, sept en Lorraine et six en Haute-Alsace ${ }^{12}$. Les sous-préfets y sont remplacés

\footnotetext{
${ }^{10}$ Par exemple en nommant en 1901 le comte Friedrich von Zeppelin président de Lorraine.

" Nachlaß Hammerstein, Bundesarchiv Coblenz, NH 24-25.

${ }^{12}$ Sans oublier que les villes d'importance - Strasbourg, Metz, Mulhouse et Colmar - sont placées sous la responsabilité spécifique d'un directeur de police (Polizeidirektor).
} 
par des »directeurs de cercle" (Kreisdirektoren) aux compétences administratives élargies, tant en milieu rural (contrôle des communes, direction des comices agricoles...) qu'urbain (attributions de police...), complétées par des pouvoirs politiques: surveillance des associations, partis politiques, journaux, de la circulation des étrangers, etc. En cela, les directeurs de cercle peuvent être tenus pour les pivots de la nouvelle administration allemande, ce qui leur vaut les attaques les plus virulentes des milieux francophiles, évoquant par exemple ces "petits autocrates, toujours présents, toujours agissant et d'autant plus abominables et intraitables qu'ils se font une idée plus haute de leur importance gouvernementale ${ }^{13}$.

Au niveau des communes, les directeurs de cercle considèrent les maires suivant le modèle du Bürgermeister prussien, c'est-à-dire comme des auxiliaires de l'administration plus que des élus du suffrage, ce qui pose problème aux élus alsaciens-lorrains notamment lorsqu'il leur est demandé de surveiller le séjour des étrangers ou les mouvements d'option vers la France ${ }^{14}$. L'interprétation allemande l'emporte définitivement en 1887 lorsqu'est votée au Reichstag la loi sur les maires de carrière (Berufsbürgermeister): le texte permet d'imposer au conseil municipal élu un maire extérieur nommé, en particulier dans les villes, où sont fréquemment affectés de jeunes fonctionnaires allemands, et ce jusqu'en $1918^{15}$.

\section{"Gut verwaltet aber schlecht regiert ${ }^{16}$ \\ Une administration d'entre-deux}

L'administration du Reichsland et ses fonctionnaires apparaissent fondamentalement marqués par toute une série de dualismes, qui renvoient à la situation singulière de la terre d'Empire elle-même: inédite, fluctuante et contrainte de l'extérieur, loin de toute image monolithique.

${ }^{13}$ Brochure L'Alsace-Lorraine et l'Empire germanique, citée par ROTH, La Lorraine annexée, p. 75.

${ }^{14}$ Voir par exemple Henri HiEgel, L'option et l'émigration dans l'arrondissement de Sarreguemines, dans: Annuaire de la Société d'histoire et d'archéologie lorraine (1974).

is Archives départementales de la Moselle, 10 AL 88-89; Statistisches Büro des Ministeriums für Elsaß-Lothringen, Das Reichsland Elsaß-Lothringen, Landes- und Ortsbeschreibung, vol. 1, Strasbourg 1898, p. 217; et Robert WUTTKE, Die Deutschen Stădte, Leipzig 1904 , p. 15.

${ }^{16}$ On renvoie ici à la formule d'Otto MEISSNER, Elsaß und Lothringen als Reichsland, 18711918, dans: Paul WentzKe (dir.), Elsaß und Lothringen, deutsches Land, Berlin 1941 (Meißner est alors secrétaire d'État à la présidence du Reich). 


\section{Dyarchies au sommet}

Ceci est particulièrement net durant les premières années de l'annexion, marquées par le régime de la Présidence supérieure, qui porte en lui la gestion des affaires à deux échelons, Berlin et Strasbourg. Les instances berlinoises se sont vu confier la réalité du pouvoir, à travers l'office d'Alsace-Lorraine à la chancellerie. Par ces bureaux, Bismarck contrôle les secteurs administratifs dévolus à la compétence exclusive de l'Empire: chemins de fer, postes, université, mines et forêts... Il assume également la responsabilité constitutionnelle, prépare les projets de loi et les soumet aux chambres, Bundesrat et Reichstag. Dans la pratique, c'est un haut fonctionnaire, Karl Herzog, pendant dix ans à la tête de l'office d'Alsace-Lorraine, qui exerce ici une influence d'importance. Ce dernier trouve face à lui, à Strasbourg, un président supérieur (Oberpräsident), haut fonctionnaire lui aussi, qui représente l'Empereur dans le Reichsland et dispose en principe d'attributions larges - administration intérieure, commerce et industrie, agriculture, finance, etc. -, mais en position d'exécutant, sous la tutelle permanente des services de Berlin. Or, le président supérieur Eduard von Moeller n'est pas homme de compromis. D'origine prussienne, il peut se prévaloir en tant que président supérieur de HesseNassau après 1866 d'avoir réussi à intégrer dans la Prusse cette conquête, et a depuis ses entrées auprès de Guillaume $\mathrm{I}^{\text {er }}$. Aussi entre-t-il en conflit permanent avec Herzog lorsque celui-ci cherche à le contrôler ou amender ses vues sur les politiques de germanisation. Certes, Moeller arrive à faire accepter en 1874 au chancelier la création du LandesausschuB, repoussée en 1871, y voyant une étape pour consolider les "faits accomplis« en direction de l'autonomie ${ }^{17}$. Mais, en mars 1878 , Herzog est promu au grade de secrétaire d'État à la chancellerie, désormais intermédiaire obligé entre Moeller et Bismarck. Pour contourner ce problème de personnes, Moeller lance l'idée de transformer le Reichsland en Kronprinzland, une principauté gouvernée par le prince impérial, mais ne rencontre pas d'écho favorable auprès de ce dernier ni de l'Empereur, pas plus du reste du côté des élus de la Délégation ${ }^{18}$. Son sort est alors scellé avec la réforme de 1879: il doit quitter Strasbourg ${ }^{19}$.

L'introduction de la »Constitution de $1879 \lll$, si elle consacre le primat administratif de Strasbourg entre les mains d'un Statthalter, n'en finit pas avec les >dualités-duels` à la tête des services. En effet, la posture du gouverneur ne peut être comprise en dehors de ses rapports avec le chancelier à Berlin d'une

${ }^{17}$ Georg Wolfram, Oberpräsident Eduard von Möller, et les souvenirs du fonctionnaire Fritz BRONNER: Die Verfassungsbestrebungen des Landesausschusses für Elsaß-Lothringen. 1875-1911, Heidelberg 1926.

${ }^{18}$ Rudolf SCHRAMM, Kronprinzenland Elsaß-Lothringen, Mailand 1878.

${ }^{19}$ Gazette de Lorraine, 30 septembre 1879. Moeller déclare laisser au Reichsland mune administration bien ordonnée, des finances équilibrées et une représentation politique lui permettant de progresser dans une bonne voie par ses propres moyens«. 
part, et le nouveau ministère d'Alsace-Lorraine à Strasbourg de l'autre. Par rapport au président supérieur, le Statthalter cumule pour le Reichsland pouvoir souverain et pouvoir exécutif, "lieutenant impérial en terre d'Empire et chancelier responsable pour l'Alsace-Lorraine «" ${ }^{20}$ ' est donc désormais à lui que reviennent la responsabilité constitutionnelle et le contreseing des lois et décrets pour l'Alsace-Lorraine exercés depuis 1871 par Bismarck. En pratique, ce dernier ne se prive pas d'intervenir très directement pour des mutations de fonctionnaires supérieurs ou, plus largement, avant la présentation des textes de loi: par exemple, le projet de loi municipale a été ajourné par Bismarck en 1889 en vue d'un examen par les ministères prussiens; il n'est revenu au Statthalter qu'en 1894 pour être déposé au Landesausschu $\beta^{21}$. La fonction de Statthalter peut ainsi sembler contradictoire en elle-même, dans la mesure où son titulaire est censé être en Alsace-Lorraine tout à la fois "l'alter ego de l'empereur souverain « - c'est-à-dire bien plus qu'un fonctionnaire - et »un simple ministre impérial« soumis aux consignes de la chancellerie ${ }^{22}$. La marge d'initiative du Statthalter tient aux contacts directs qu'il peut avoir avec l'Empereur et son entourage proche par le système de l'Immediatbericht: les décisions d'importance circulent ainsi entre la chancellerie, le cabinet civil de l'Empereur et le Statthalter, qui ne parvient jamais dans les faits à se maintenir bien longtemps contre l'avis du chancelier: Hermann von Hohenlohe-Langenburg, par exemple, a été poussé à la démission en 1907 pour avoir déplu au chancelier Bernhard von Bülow ${ }^{23}$.

Parallèlement, la réforme de 1879 transfère à Strasbourg les services administratifs jusque-là attachés à la chancellerie, avec à leur tête le secrétaire d'État Karl Herzog qui prend la direction du Ministerium. Subordonné et suppléant du Statthalter, il a pour fonction de diriger l'administration, composée de quatre sections: intérieur, justice et cultes, finances, commerce et travaux publics, relevant chacune d'un sous-secrétaire d'État. Mais en même temps, il dispose de prérogatives propres: les relations avec l'Empire et la correspondance avec les autorités de Berlin, les rapports avec le Landesausschuß et le Bundesrat, ou encore les dossiers personnels des fonctionnaires supérieurs du Reichsland. On le comprend, le fonctionnement des institutions de Strasbourg suppose une collaboration étroite entre le Statthalter et le secrétaire d'État, dont les compétences respectives ne sont pas strictement définies et encore moins disjointes ${ }^{24}$. Ce >duel-duor révèle l'indétermination qui prévaut jusqu'au plus haut niveau: l'entregent fait souvent office d'arbitre et chaque res-

\footnotetext{
${ }^{20}$ ROTH, La Lorraine annexée, p. 62.

${ }^{21}$ Deutsches Zentralarchiv (DZA) Potsdam RK 150, 164 et 190.

22 Jean-Marie Mayeur l'a bien souligné: voir ID., Autonomie et politique en Alsace, p. 14.

${ }^{23}$ DZA RK 159.

${ }^{24}$ Le Statthalter Manteuffel le fait remarquer dans un rapport à l'Empereur du 12 janvier 1880: DZA RK 157.
} 
ponsable apparaît en permanence sous le regard d'un autre. Malgré les critiques des Statthalter vis-à-vis de ce système dual ${ }^{25}$, il reste en vigueur jusqu'en 1918 et explique l'importance des facteurs personnels dans la direction du Reichsland.

De 1879 à 1914, la terre d'Empire est administrée par quatre Statthalter successifs, dont la personnalité se répercute sur le fonctionnement des services: Edwin von Manteuffel (1879-1885), Chlodwig zu Hohenlohe-Schillingsfürst (1885-1894), Hermann von Hohenlohe-Langenburg (1894-1907) et Carl von Wedel (1907-1914). Le maréchal Manteuffel arrive à Strasbourg en 1879, à 70 ans, après une carrière militaire et diplomatique de premier plan. L'empereur Guillaume $\mathrm{I}^{\text {er }}$ a même songé un temps à lui proposer la chancellerie, si bien que les relations avec Bismarck étaient empreintes de méfiance réciproque, et c'est pour le surveiller que le chancelier lui a adjoint le secrétaire d'État Herzog. Il ne faut pas un an pour que les tensions éclatent entre Herzog, fonctionnaire rigide, et Manteuffel qui, au contraire, prend le parti d'ancrer les "faits accomplis« en cherchant une certaine popularité et des contacts réguliers avec les notables et le clergé, proposant aux membres de la Délégation d'arriver ainsi, à terme, à »la reconnaissance de la pleine et légitime indépendance constitutionnelle de l'Alsace-Lorraine dans l'Empire ${ }^{26}$. Ces gages aux milieux alsaciens-lorrains heurtent Herzog, rapidement démis. Le nouveau secrétaire d'État Karl von Hofmann se plaît alors prudemment dans une position d'exécutant des directives du Statthalter, lorsque ce dernier se marque dans l'ouverture ou, plus tard, se ravise vers une germanisation plus ferme contre les "protestataires« francophiles ${ }^{27}$. Il est vrai que Manteuffel ne fournit pas de consignes claires à ses subordonnés, réagissant, selon ses propres termes, en fonction de son sintuition « et de son sinstinct politique ${ }^{28}$, dans la mesure où ses connaissances administratives apparaissent limitées. Le maréchal écrit d'ailleurs à Bismarck le 28 juillet 1879: "Meine Verhältnisse haben es nun mit sich gebracht, daß ich der heutigen Beamtenwelt ziemlich fremd geworden bin ${ }^{29}$. Pour autant, le Statthalter n'accepte pas de remarques de la part des fonctionnaires du ministère ou des présidences de district: le soussecrétaire d'État Albert von Pommer-Esche et le président de Lorraine Adalbert von Flottwell sont contraints à la démission, tandis que le nouvel arrivant Hofmann se voit demander par Manteuffel - et ce n'est pas le moindre des signes de tensions au sein de l'administration de Strasbourg - de "ssurveiller

${ }^{25}$ Le Statthalter Hohenlohe parle le 25 mars 1887 d'une »erreur de premier ordre« dans une note au chancelier: DZA RMI 16713.

${ }^{26}$ Discours reproduit dans la Gazette de Lorraine, 18 décembre 1879.

${ }^{27}$ Voir le récit de l'épouse d'un des hauts responsables du Reichsland: Alberta von PuTTKAMER, Die Aera Manteuffel. Federzeichnungen aus Elsaß-Lothringen, Stuttgart, Leipzig 1903; et DZA RK $159 / 4$ et 164.

${ }^{28}$ Manteuffel à Bismarck, 7 décembre 1879, DZA Potsdam, Reichskanzlei (07.01), Nr. 157.

${ }^{29}$ Geheimes Staatsarchiv Berlin-Dahlem: Rep. 92 Nachlaß (NL) Manteuffel Nr. 43 fol. 6. 
attentivement" (gehörig überwachen) les hauts fonctionnaires, y compris hors service $^{30}$. $» E r$ hinterließ eine tief verunsicherte deutsche Beamtenschaft, eine Verwaltung in Chaos und Unordnung und ohne klar erkennbares Ziel. Der falsche Mann am falschen Ort, conclut Hermann Hiery ${ }^{31}$.

Le successeur de Manteuffel, décédé en juin 1885, est l'ambassadeur d'Allemagne à Paris, le prince von Hohenlohe-Schillingsfürst ${ }^{32}$. Ce diplomate a auparavant servi à la fois la Prusse et la Bavière et noué des relations avec Bismarck. Dans cette posture bien assise, il se présente comme un dilettante, qui ne s'investit pas spécialement en direction des milieux "indigènes ${ }^{33}$ et laisse d'abord opérer le secrétaire d'État Hofmann. Or, ce dernier s'en tient à la position en retrait qu'il occupait auprès de Manteuffel. Les élections de 1887, marquées par la protestation militante après la poussée boulangiste en France, mettent alors l'administration en difficulté. Hohenlohe-Schillingsfürst réussit à se maintenir en incriminant auprès de Bismarck la responsabilité de Hofmann, "un brave homme faible«, si bien que le chancelier le remplace au motif de sa "passivité devant la situation critique dans laquelle nous nous trouvons ${ }^{34}$. Dès lors, c'est un nouveau secrétaire d'État qui prend l'administration du Reichsland en main: Maximilian von Puttkamer, apparenté à l'épouse de Bismarck, est désigné par ce dernier pour être »un fonctionnaire prussien de tradition prussienne« et doté d'expérience; il a attesté sa rigueur et peut prétendre à une bonne connaissance du système administratif par ses précédentes fonctions dans le Reichsland: conseiller à la cour d'appel de Colmar de 1871 à 1877 , avocat général de 1877 à 1880 et sous-secrétaire d'État à la Justice de 1880 à 1887 . De fait, Maximilian von Puttkamer va tenir activement et fermement sa charge pendant presque quinze ans, de 1887 à $1901^{35}$.

En effet, le Statthalter Hohenlohe-Schillingsfürst, qui se contente de ses fonctions de représentation, est nommé chancelier d'Empire par Guillaume II en 1894. Il parvient alors à imposer à Strasbourg son cousin, HohenloheLangenburg, un militaire puis parlementaire qui n'a guère de connaissances pratiques d'administration, si bien que $\mathrm{M}$. von Puttkamer est confirmé dans ses fonctions »élargies« de secrétaire d'État. Langenburg se pose comme représentant de l'Empereur, pratiquant une libéralisation lente du régime, et non comme responsable de l'administration du Reichsland. En 1902, Emst Mathias von Koeller succède à M. von Puttkamer avec un profil similaire de rigueur, après avoir été sous-secrétaire à l'Intérieur: dirigeant les fonctionnaires

${ }^{30}$ DZA, NL Hofmann, Nr. 36 fol. 15. (21 juin 1882)

"HIERY, Reichstagswahlen im Reichsland, p. 77.

${ }^{32}$ Un portrait dans la Straßburger Post, 17 juillet 1904.

${ }^{33}$ Sont ainsi nommés durant l'annexion les Alsaciens-Lorrains »de souche " par opposition aux nimmigrés « allemands issus du Reich.

${ }^{34}$ DZA RK, 7 mars 1887.

${ }^{35}$ Straßburger Post, 17 juin 1901. 
d'une main de fer, ce conservateur prussien laisse sa marque dans ce que l'on a appelé le wrégime Koeller«: statu quo institutionnel, répression des mouvements francophiles et socialistes. De fait, lorsque Bülow remplace HohenloheSchillingsfürst à la chancellerie, Langenburg perd un appui de poids et ne peut que subir les récriminations du nouveau Kanzler qui juge son action "molle et insuffisante«. »De capacités restreintes, il règne et ne gouverne pas«, résume Bernard Vogler à propos de Langenburg ${ }^{36}$. Bülow l'écarte finalement sans ménagement; Langenburg s'en plaint d'ailleurs: „Eine brutale Absetzung. Wie einen Schurken jagt man mich fort nach dreizehnjährigen treuen Diensten ${ }^{37}$.

En novembre 1907 arrive l'ambassadeur Carl von Wedel aux fonctions de Statthalter, et le balancier repart en faveur du gouverneur et au détriment du secrétaire d'État: se voulant ouvert, Wedel s'engage de façon très active dans la question constitutionnelle qui débouche sur la réforme de 1911. Divergeant sur ces évolutions, le secrétaire Koeller est remplacé dès 1908. La succession est particulièrement significative du conflit entre les deux hommes, puisque le nouveau secrétaire d'État personnifie le pari du ralliement des notabilités alsaciennes-lorraines: il s'agit du grand propriétaire de Saverne Hugo Zorn von Bulach, pourtant considéré comme "peu sûr" par les milieux pangermanistes $^{38}$.

\section{La coexistence conflictuelle de trois fonctions publiques dans le Reichsland}

Le mode de fonctionnement dual n'est pas propre au sommet de la terre d'Empire, bien au contraire: s'il marque l'ensemble de l'administration, c'est notamment en raison des différents corps auxquels est confié le pouvoir, sans répartition toujours claire. De là découlent deux césures parmi les fonctionnaires en poste dans le Reichsland: entre fonctionnaires de l'Empire et de la terre d'Empire d'une part, entre fonctionnaires civils et militaires de l'autre.

En effet, parmi les fonctionnaires civils affectés dans le Reichsland coexistent deux catégories bien différentes: les Reichsbeamten et les Reichslandsbeamten. Les premiers sont affectés dans les administrations relevant directement de l'Empire: chemins de fer, postes, douanes..., tandis que les seconds remplissent les services territoriaux, car l'Alsace-Lorraine, quoique non reconnue comme État confédéré, possède un service public propre, le Reichslandsdienst, relevant hiérarchiquement de l'Empereur via un serment de fidélité, et dans la pratique du pouvoir de nomination du Statthalter. Le départ après 1871 de nombreux fonctionnaires vers la France a permis une germanisation

\footnotetext{
${ }^{36}$ VOGLER, Histoire politique de l'Alsace, p. 179.

${ }^{37}$ Note de journal intime, 6 octobre 1907, Eißele, 1950, p. 162.

${ }^{38}$ Archives départementales du Bas-Rhin (ADBR), NL Bulach Nr. 24.
} 
de ces fonctions, mais en même temps c'est parmi ces Reichslandsbeamten que l'on trouve un nombre non négligeable d'Alsaciens (plus que de Lorrains), en particulier aux postes inférieurs ${ }^{39}$.

Plus conflictuel encore est le rapport entre les administrateurs civils et militaires, à tout le moins après le décès, en 1885, du maréchal Manteuffel, premier et seul Statthalter à cumuler les deux domaines de compétences. Les relations avec les commandants des corps d'armée sont ensuite marquées par une tension latente, d'autant plus que ces demiers dépendent du ministre de la Guerre à Berlin et du cabinet militaire de l'Empereur. Les fonctionnaires civils se retrouvent alors fréquemment débordés, y compris dans la hiérarchie des préséances. Par exemple, en Lorraine, la création du XVI ${ }^{\mathfrak{e}}$ corps d'armée s'est traduite par l'affectation de généraux de premier rang; quatre ont même été gratifiés du titre suprême dans l'administration, "Exzellenz« - ce qui n'est le cas d'aucun fonctionnaire civil du Bezirk, pas même le président ${ }^{40}$. Cette ninfériorité« des civils en cas de divergence se laisse particulièrement remarquer dans les années 1910 et la montée des tensions franco-allemandes qui conforte l'autorité de l'état-major sur la marche de l'Est (Westmark). Le Statthalter von Wedel en fait les frais après l'»affaire de Saverne« en 1913: un jeune lieutenant s'en est pris à des recrues alsaciennes, ce qui a provoqué une forte émotion dans la région. Les responsables militaires en tirent parti pour faire tomber le secrétaire d'État »rallié« Zorn von Bulach. Quelques semaines plus tard, Wedel lui-même est renvoyé par Guillaume II, en même temps que ses principaux collaborateurs du Ministerium: c'est le »Regierungswechsel in Straßburg «. Les militaires reprochent en effet à l'ensemble des hauts responsables civils leur inaction face aux manifestations patriotiques françaises: par exemple, en 1913, le président de police de Mulhouse s'est retrouvé dans la tourmente après qu'un orchestre suisse a joué "La Marseillaise « dans un café de la ville; Wedel avait alors pris sa défense contre les demandes de radiation lancées par les généraux. En 1914, les nouveaux administrateurs civils sont choisis pour leur fidélité à l'Obrigkeitsstaat, à commencer par le Statthalter Johann von Dallwitz, jusque-là ministre de l'Intérieur de Prusse, un conservateur qui veut »imposer les recettes de la froideur impersonnelle « ${ }^{4 !}$.

\footnotetext{
${ }^{39}$ Philippe GERBER, L'administration en Alsace-Lorraine, dans: Revue de droit public et des sciences politiques 4 (1909) p. 1-24.

${ }^{40}$ ROTH, La Lorraine annexée, p. 73.

4 ' VOGLER, Histoire politique de l'Alsace, p. 179 et 211; David SCHOENBAUM, Zabern 1913. Consensus Politics in Imperial Germany, Londres 1982.
} 
Les carrières des fonctionnaires allemands du Reichsland se fondent également sur des dualités qui ont leur origine dans la formation singulière de l'Alsace-Lorraine. Si les règles de fonctionnement de l'administration de la terre d'Empire ont été largement inspirées de celles en vigueur en Prusse, cette origine a été dans la pratique »acclimatée« au contexte particulier pour ce qui touche à la gestion des personnels intermédiaires et supérieurs, qui suit une véritable hiérarchie, dont la progression apparaît réglée par des mouvements entre territoires.

\section{Le cursus honorum administratif en terre d'Empire}

Schématiquement, la fonction publique du Reichsland se décompose en trois grades: stagiaire ou référendaire (Referendar), assesseur (Assessor) et conseiller de gouvernement (Regierungsrat). Le parcours des différents fonctionnaires suit ce modèle, mais selon des déclinaisons variables en fonction des affectations effectives. La première »étape «, celle de stagiaire, correspond à la sortie de l'université: une fois sa demande validée, l'aspirant accomplit sept stages d'une durée totale minimale de trois ans et demi, pour lesquels il n'est guère rémunéré, et rédige un mémoire sur un sujet imposé, avant de passer l'examen d'État (Staatsprüfung) et d'être enfin reconnu apte à occuper un poste dans l'administration. Ce schéma correspond à une triple sélection successive: universitaire, financière et politique.

Pour les reçus commence alors l'assessorat. Deux types de fonctions peuvent être distinguées ici par rapport aux avancées de carrière: assesseurs de cercle, respectivement de présidence et de ministère; les seconds, plus rares, accèdent bien plus rapidement à la titularisation ${ }^{42}$ et au grade de conseiller. François Roth donne les exemples suivants: d'une part, le baron bavarois von Bibra, entré dans l'administration du Reichsland en 1873 à 31 ans, occupe cinq postes en neuf ans comme assesseur de cercle (Kreisassessor) - Boulay, Ribeauvillé, Altkirch, Thionville et Colmar -, avant de devenir directeur du cercle de Boulay en 1883 à 41 ans; de l'autre, Wilhelm Back, fils du maire allemand de Strasbourg, est davantage favorisé dans son assessorat, car après un premier poste dans l'important Kreis de Mulhouse, il est affecté aux présidences de district de Metz et Strasbourg, puis même au secrétariat à la Justice à Berlin: à 34 ans seulement, il accède ensuite à la direction de cercle de Château-Salins.

${ }^{42}$ Le statut des assesseurs est flou: certains postes sont inscrits au budget général, d'autres pas; certains sont fonctionnaires titulaires, d'autres non. 
Les conseillers de gouvernement sont également répartis en deux groupes, de valorisation différente: les directeurs de cercle, d'une part, les conseillers de présidence ou de ministère, de l'autre. Là encore se dessine un vrai parcours, au sein même de ces deux pôles, et le plus souvent très progressif: dans le premier cas, directeur de cercle rural (Boulay, Château-Salins, Haguenau, Erstein...) puis de cercle urbain (Thionville, Mulhouse, Metz ou Strasbourg), enfin président de district; dans le second, conseiller de présidence, conseiller spécialisé, conseiller supérieur, "véritable et intime" (Wirklicher Geheimer Regierungsrat), enfin; fort rarement, excellence (Exzellenz). Une distinction importante entre ces deux profils et leurs évolutions tient à la stabilité ou non dans les fonctions exercées. Les conseillers de présidence s'inscrivent dans la durée; par exemple, en Lorraine, le conseiller spécialisé à l'industrie Rick (Gewerberat) est resté en poste à Metz pas moins de 27 ans, de 1891 à 1918. Ce type de postes constitue une fin de carrière valable et permet une promotion sans changement de fonction (devenir in fine conseiller "véritable et intime $\ll)^{43}$. À l'inverse, les directeurs de cercle tournent assez rapidement: on en compte entre sept et dix dans les différents Kreise de 1871 à 1914, et seul un petit nombre acquiert une notoriété, et encore hors du circuit administratif "classique«, à l'exemple d'Otto Poehlmann à Sélestat qui réussit en 1893 à se faire élire député de la circonscription. De même, la rotation est importante au niveau supérieur: on dénombre entre sept et onze présidents pour chaque $B e$ zirk de 1871 à 1914, avec une durée parfois réduite à quelques mois ${ }^{44}$. Ces mouvements peuvent tenir à l'organisation même d'un cursus honorum, mais s'expliquent surtout plus spécifiquement en fonction de contingences propres à la terre d'Empire, qui dépend de fait de Berlin et associe de façon inédite et artificielle Alsace et Lorraine.

\section{Une double dualité des carrières en écho aux divisions territoriales}

Deux traits retiennent l'attention lorsqu'on examine les évolutions de carrière des fonctionnaires occupant des postes moyens ou élevés dans le Reichsland: la primauté des "parachutages « de fonctionnaires prussiens sur les promotions des administrateurs allemands d'Alsace-Lorraine et la prééminence, dans la hiérarchie interne, des fonctionnaires exerçant en Alsace sur ceux affectés en Lorraine.

Les nominations directes de fonctionnaires prussiens ont été particulièrement nombreuses durant les premières années de l'annexion, dans l'objectif d'une politique de germanisation qui ne soit érodée par l'habitude prise des contingences locales. Mais ces pratiques continuent de s'exercer par la suite

\footnotetext{
${ }^{43}$ Voir ROTH, La Lorraine annexée, p. 67-69.

${ }^{44}$ VOGLER, Histoire politique de l'Alsace, p. 198-200.
} 
pour des postes élevés, à partir de réseaux dans les ministères de Berlin, au détriment des agents sur place. Ainsi, sur les onze présidents de Lorraine, sept sont d'origine prussienne: Guido Henckel von Donnersmarck (août 1870-janvier 1871), Botho von Eulenburg (janvier 1872-mars 1873), Adolf von Amim (mars 1873-octobre 1874), Robert von Puttkamer (janvier 1875-mars 1877), Friedrich von Reitzenstein (mars 1877-avril 1880), Adalbert von Flottwell (avril 1880-août 1883) et Hans von Hammerstein (août 1883-mars 1901). Plus clairement encore, cinq d'entre eux sont issus de la noblesse administrative et militaire de Prusse: von Eulenburg, von Arnim, Robert von Puttkamer, von Flottwell et von Reitzenstein. Ainsi, l'administration du Reichsland est d'abord dévolue aux fonctionnaires prussiens, bien plus qu'aux autres États confédérés, malgré le principe du statut de terre d'Empire. Également riche de sens est la nomination par Bismarck, en 1875, de Robert von Puttkamer, président de Prusse orientale, à la présidence de Lorraine, alors que le président supérieur Moeller avait préconisé l'avancement du conseiller von Reitzenstein, en poste à Metz depuis 1871 et qui a assuré l'intérim. Ce n'est qu'au départ de Robert von Puttkamer que ce dernier accède à la présidence, alors qu'il est, lui aussi, originaire de Prusse. C'est la filière "Prusse-Reichsland et retour« qui est privilégiée pour les progressions de carrières, plutôt que les promotions sur place. Trois décennies plus tard, le fils de Robert von Puttkamer est envoyé directement de Prusse à la présidence de Haute-Alsace ${ }^{45}$. Ce schéma explique le nombre important de présidents de district qui se succèdent en un temps assez bref: pour les intéressés, c'est là le meilleur moyen d'obtenir rapidement une promotion entre le grade de Landrat, sorte de sous-préfet en Prusse, et celui de président supérieur (Oberpräsident). Par exemple, Arnim quitte Metz au bout de 18 mois pour la présidence supérieure de Westphalie, et R. von Puttkamer pour celle de Silésie. Le prix à payer est l'éloignement, et Eulenburg, Arnim comme Robert von Puttkamer n'ont de cesse de solliciter des demandes de congé, d'autant plus que ces proches de la Cour sont simultanément Bezirkspräsident à Metz et députés au Landtag de Prusse ou au Reichstag... Le passage par la terre d'Empire est un accélérateur de carrière qui permet à tous les trois de terminer leur carrière comme ministre en Prusse $^{46}$.

Cette prépondérance prussienne se vérifie également à l'échelon inférieur des directeurs de cercle. En Lorraine par exemple, 45 des 54 directeurs entre 1871 et 1914 sont prussiens, soit tout de même $85 \%$. La différence vient de ce que, à ce niveau, le passage par le Reichsland ne suffit pas en soi pour espérer une promotion directe et rapide en Prusse: de Lorraine, seul l'un d'entre

\footnotetext{
${ }^{45}$ Moniteur de la Moselle, 14 novembre 1874; ADBR, 87 AL (4807).

46 ADBR, AL 87 (3428); Alberta von PUTTKAMER, Staatsminister Robert von Puttkamer, Leipzig 1928; ROTH, La Lorraine annexée, p. 77-78.
} 
eux, Paul Boehmer, y devient sous-secrétaire d'État aux colonies ${ }^{47}$. Pour les autres, c'est une seconde voie qui s'ouvre: l'avancement en Alsace.

$\mathrm{Au}$ sein de la terre d'Empire, les passages de fonctionnaires d'Alsace en Lorraine et réciproquement sont significatifs de leur avancée de carrière, et révèlent les processus de constitution du territoire nouvellement annexé entre deux États: l'attrait de l'Alsace et de Strasbourg est réel dès l'origine pour des raisons historiques de proximité plus grande avec les pays germaniques, tandis qu'une bonne partie du département de Moselle est, au XIX ${ }^{\mathrm{e}}$ siècle, de langue et de culture françaises, à commencer par la ville de $\mathrm{Metz}^{48}$. L'accession de Strasbourg en 1879 au rang de capitale provinciale avec la mise en place du Statthalterat accélère encore le phénomène: pour ceux qui ne parviennent pas à trouver une promotion dans leur État d'origine, c'est en Alsace que leur carrière peut se terminer de façon acceptable, tandis qu'un passage en Lorraine est l'occasion d'avancer dans le rang des fonctions remplies. Ce rôle-étape d'une affectation messine pour favoriser la progression d'un fonctionnaire est largement vérifié. Par exemple, Alexander Halm, maire de Metz de 1880 à 1895, devient ensuite président du Bezirk de Basse-Alsace de 1895 à 1908. Le directeur de cercle de Thionville Cordemann termine son service à Strasbourg comme directeur de l'»Office des assurances du Reichsland (Landesversicherungsanstalt). Quant au directeur de cercle de Forbach de 1889 à 1896, Albert Dieckmann, après être promu en Alsace comme directeur du cercle de Sélestat, il devient président de police de Mulhouse et directeur de l'Office des assurances après Cordemann. De la même façon, le baron von Hammerstein, directeur des importants cercles alsaciens de Colmar puis Mulhouse, passe par la Lorraine pour atteindre le grade plus rare de Bezirkspräsident: il est affecté comme président à Metz de 1883 à 1901. Quant aux fonctionnaires lorrains de cercles, leur promotion de fin de carrière est, de façon significative, synonyme de poste en Alsace: de 1871 à 1914, sur les seize fonctionnaires qui ont atteint le grade de conseiller supérieur, treize ont été affectés en récompense à la capitale régionale Strasbourg ${ }^{49}$.

Ces trajectoires montrent dans leur pluralité que le Reichsland d'AlsaceLorraine est tout sauf une unité autonome, une construction de circonstances ouverte aux conflits régionaux, nationaux et internationaux, et les fonctionnaires allemands sont spécialement pris dans ces tensions. En effet, le cadre périphérique sui generis de la terre d'Empire favorise le développement d'une économie d'enracinement qui repose sur des références spécifiques, de nature

\footnotetext{
${ }^{47}$ ADBR, AL $69(168,210,262,316)$.

${ }^{48}$ Voir Serge BONNET, Sociologie politique et religieuse de la Lorraine, Paris 1972; Henri CONTAMINE, Metz et la Moselle de 1814 à 1870, Nancy 1932; François ROTH, Les Lorrains entre la France et l'Allemagne, Metz, Nancy 1981.

${ }^{49}$ Note sur la carrière, les titres et le rang des fonctionnaires en Alsace-Lorraine, p. 734-755, citée par ROTH, La Lorraine annexée, p. 69-70 et p. 79.
} 
à exacerber la perception et la valorisation des différences ${ }^{50}$. Affectés à Strasbourg, dans les Bezirke, les Kreise comme dans les communes, ils ont les premiers à gérer les effets de l'annexion sur le plan local. Ce sont ces contradictions de l'entre-deux contraignant le degré de pénétration administrative du territoire que révèlent avec netteté les parcours et les terrains que nous avons suivis, en même temps qu'ils donnent à voir en pareilles circonstances le poids des facteurs personnels et d'interconnaissance au sein de réseaux politicoadministratifs, civils et militaires, aux contours non stabilisés, en redéfinition permanente. De la sorte, on saisit mieux sur quels personnels l'Empire s'appuie pour intégrer l'Alsace-Lorraine. Des possibilités de carrière s'y révèlent, en même temps que de nombreux va-et-vient à tous les échelons. En particulier, les contradictions au sommet traduisent des négociations permanentes entre les responsables administratifs de la terre d'Empire et le gouvernement de Berlin, et permettent d'évaluer à la fois les marges de manœuvre dont disposent les différents fonctionnaires sur place, mais aussi le poids des réseaux militaires dans la tradition administrative prussienne simportée« en AlsaceLorraine, et même un clientélisme familial autour de Bismarck. À l'image de la terre d'Empire elle-même, ses fonctionnaires apparaissent particulièrement "dépendants « de niveaux de décision et d'action multiples qui ne coïncident guère, ce qui fonde en 1914 la conviction du juriste alsacien Robert Redslob: "Elsaß-Lothringen wird nicht immer ein Besitzobjekt des Reiches bleiben ${ }^{51}$.

\section{Deutsche Zusammenfassung}

Bei der Frage nach den Figurationen des Staates in Frankreich und Deutschland bietet es sich an, die einzigartige historische Konstruktion des Reichslandes Elsaß-Lothringen zwischen 1871 und 1918 genauer zu betrachten. Interessant ist hier vor allem die Einrichtung einer neuen, deutschen Verwaltung in der annektierten Zone. In diesem Sinne analysiert der Beitrag die gängigen Diskurse über die Germanisierungspolitik, indem er die personelle Verkörperung und Entwicklung des deutschen Staates im Reichsland aufzeigt.

Um den Aufstieg der »importierten« Eliten, die zwischen administrativer Kontinuität und Erneuerung, Bürokratie und Politisierung schwanken, besser zu verstehen, konzentriert sich die Betrachtung auf das Profil und die Tätigkeit der Beamten, die auf verschiedenen Ebenen staatlicher Intervention eingesetzt wurden. So wird verständlich, auf welches Personal sich das Kaiserreich stützte, um das eroberte Gebiet zu durchdringen, und welche Berufsmöglichkeiten sich hier eröffneten. Weiterhin werden die Gegensätze, die auf höchster staatlicher

${ }^{\text {so }}$ Sur le plan des représentations, voir notre article: Philippe HaMMAN, Une entreprise de mobilisation patriotique: la production de la faïencerie de Sarreguemines (1871-1918), dans: Genèses. Sciences sociales et histoire 47 (2002) p. 140-161. Par analogie, voir aussi quant à la frontière franco-belge, Michel HASTINGS, Identité culturelle locale et politique festive communiste: Halluin-la-Rouge. 1920-1934, dans: Le Mouvement social 139 (1987) p. 7-25.

s1 "L'Alsace-Lorraine ne restera pas toujours un objet en possession de l'Empire«: REDSLOB, Unabhängige Länder, p. 67. 
Ebene bei der Bewältigung einer bisher unbekannten Situation und bei der Verwaltung der "Zwischenstaatszone« zu lokalen Erschütterungen führten, beleuchtet.

Die Laufbahnen der Akteure und die institutionelle Dynamik liefern anhand ihrer Entwicklung wichtige Aufschlüsse über die Auswirkungen regionaler, nationaler und zwischenstaatlicher Konflikte in diesem regelrechten »Labor« der Germanisierung. 